[I226] Quality assurance issues pertaining to nun-uniform dose prescription situations

Daniela Thorwarth *

University of Tübingen, Department of Radiation Oncology, Section for Biomedical Physics, Tübingen, Germany

* Corresponding author.

Abstract. In the last years, non-uniform dose prescriptions have gained a lot of attention as they offer the potential to irradiate tumours adapted to their internal radiation sensitivity which may be locally varying. Such dose painting concepts have been proposed based on functional imaging data, which may be ideal imaging biomarkers to assess individual radiation sensitivities in a locally resolved manner.

However, to date this concept has only been applied in a small number of clinical trials which may be due to the high complexity of this approach in terms of imaging, radiation therapy planning and also quality assurance.

In this talk, quality assurance of dose painting will be discussed starting from image acquisition and analysis towards implementation of non-uniform dose optimization, dose painting treatment planning and delivery. Furthermore, some clinical examples will be given.

Dose painting has been shown to be a very elegant concept for personalized radiation therapy approaches. However, quality assurance of the whole process remains challenging.

https://doi.org/10.1016/j.ejmp.2018.06.298

\section{[I227] Physics, technology and QC of breast tomosynthesis} Anders Tingberg *

Skåne University Hospital. Malmo, Radiation Physics, Malmö, Sweden * Corresponding author.

Purpose. Breast tomosynthesis is a semi-three-dimensional breast imaging technique that has gained a large interest over the last few years. Several published studies have shown potential to increase the cancer detection rate and to reduce recall rates in breast cancer screening. This presentation aims to summarise physics, technology and quality control of breast tomosynthesis systems.

Physics and technology. A breast tomosynthesis unit is similar to a digital mammography unit, with one major difference: the X-ray tube rotates around the breast while the detector is stationary. The tube typically rotates $15-50$ degrees while $9-25$ projection images are taken. The total radiation dose for these projection images is typically about the same as for two standard mammography images (the cranio-caudal and the medio-lateral oblique projections). The projection images are reconstructed to form an image volume from which individual slice images can be studied. Contrary to computed tomography, the spatial resolution is not isotropic (i.e. the resolution in the image plane is superior to the depth resolution) due to the limited sweep angle.

A synthetic mammogram is reconstructed from the projection images, with the intent to mimic an ordinary mammogram as closely as possible. The motivation of the synthetic mammogram is to replace the ordinary mammogram in that direction to save radiation dose, while providing an overview image as a starting point in the diagnostic evaluation for the breast radiologist.

Quality control. Quality control in breast tomosynthesis includes the tests that are done for digital mammography, but also tests that are unique to tomosynthesis, i.e. control of the movement of the Xray tube and the exposures during the tomosynthesis sweep, and the image quality of the reconstructed images.

https://doi.org/10.1016/j.ejmp.2018.06.299
[1228] Breast tomosynthesis: Technology, quality control, and dosimetry

Ioannis Sechopoulos*

Radboud University Medical Center, Department of Radiology and Nuclear Medicine, Dutch Expert Centre for Screening, Nijmegen, Netherlands

* Corresponding author.

The introduction of digital tomosynthesis to breast imaging has brought about the potential for a substantial improvement in breast cancer detection and diagnosis. However, the limited angle nature of tomosynthesis acquisitions makes this modality susceptible to image artifacts. In addition, questions on dose are especially important when the modality is or has the potential to be used for screening of asymptomatic patients. In addition, the uncertainty on how digital tomosynthesis will be used in the long term, i.e. as a replacement, adjunct, or some combination thereof of mammography, makes the dosimetric concerns more complicated. We will review the basic concepts of breast tomosynthesis, the current technology used for this modality, discuss its current dosimetric characteristics, and present the current issues and upcoming solutions in quality control of tomosynthesis systems.

https://doi.org/10.1016/j.ejmp.2018.06.300

[1229] Refresher course: Quality and safety management of medical lasers

Alex Rem*

University Medical Center, Utrecht, Netherlands

* Corresponding author.

Medical lasers have become common medical tools with their main use of cutting and coagulating tissue. Laser techniques are being used for very different medical procedures and by a wide variety of medical specialties. The techniques are very useful but may also both pose a risk to the patient, the surgeon and the workers in de room.

In this refresher course we present basic knowledge on the mechanism of action for different treatments. We will explain the interaction of laser light with tissue and other materials and Schlierenvisualizations of the effects. Most common medical lasers will be explained, such as CO2 laser, KTP-laser, Holmium laser, Nd:YAG and Ho:YAG lasers.

Main focus points for optimal use of lasers in the clinical situation will be presented. On the other hand, we will also show several situations that may cause a hazardous situation. Recently hospitals have focused on competency levels and core knowledge required by medical users; we will present the current status. Highlights of European and local standards that describe laser safety rules and organizational laser safety structure are recapitulated.

https://doi.org/10.1016/j.ejmp.2018.06.301

\section{[1230] Safe use of electrosurgery in healthcare} Christiaan van Swol ${ }^{*}$

\section{St Antonius Hospital, Medical Physics, Nieuwegein, Netherlands}

* Corresponding author.

Purpose. Nowadays, it is impossible to imagine hospitals without medical technology. Many devices have become part of everyday practice, such as electrosurgical units. Electrosurgery uses electricity to gain a surgical effect. The risk of working with this technique is 
often underestimated by healthcare professionals. This makes the technique potentially hazardous for patient, surgeons and assistants. A safe and effective use of the technique requires theoretical knowledge and practical skills. In this abstract, we describe the initiative to formulate competence requirements for using electrosurgery that are general applicable and can be used as input for training and examination.

Methods. A group of medical physicists located in eight Dutch hospitals used educational methods to analyze the use of electrosurgery in the operation room and determine competence requirements. Additionally, the group investigated resources like the Dutch risk profile for electrosurgery, risk analyses of individual hospitals, supplier manuals, user protocols, (nearly) incident reports and available local training materials. For each requirement, an explanation was given and it was pointed out if the requirement is verifiable in practice. The result was presented to users of electrosurgery such as urologists and surgeons. Furthermore, a member consultation of the association of general medical physicists took place. Outcomes of consultations were used to improve and complete the requirements.

Results. The final requirements are clustered in electricity, technology, tissue effect, instruments and their use, settings, minimal invasive surgery, risks (patient electrode patches, alternative current flow, fire, long term use, contamination of electrode) and surgical smoke. It appeared to be important for physicians to have a good perception of the behavior of electrical current in the human body, without elaborating in too much physics and mathematical equations. Best results are obtained applying a combination of theoretical explanation followed by practicing in a skills lab.

Conclusions. Safe patient care requires qualified medical staff. The approach which combined physical knowledge and educational methods successfully resulted in competence requirements. These requirements are used by hospitals as input for teaching materials and to state a minimum level of qualification for using electrosurgery safely and effectively in patient care.

https://doi.org/10.1016/j.ejmp.2018.06.302

[I231]

Abstract not available

[I232] Radiomics: Transforming standard imaging into mineable data related to biology

Philippe Lambin*

Maastricht University, The D-Lab/Radiotherapy-Um, Maastricht, Netherlands

* Corresponding author.

Introduction. The rise of radiomics, the high-throughput mining of quantitative image features from (standard-of-care) medical imaging for knowledge extraction and application within clinical decision support systems (animation: https://youtu.be/Tq980GEVP0Y) to improve diagnostic, prognostic, and predictive accuracy, has significant and substantial implications for the medical community $(1,2$, 5). Radiomic analysis exploits sophisticated image analysis tools and the exponential growth of medical imaging data to develop and validate powerful image-based signatures/models.

Materials and methods. We will describe the process of radiomics, its pitfalls, challenges, opportunities, and its capacity to improve clinical decision making (presently primarily in the care of patients with cancer, however, all imaged patients may benefit from quantitative radiology) $(5,8)$. Finally, the field of radiomics is emerging rapidly; however, the field lacks standardized evaluation of both the scientific integrity and the clinical significance of the numerous published radiomics investigations resulting from this growth.

Results. There is a clear and present need for rigorous evaluation criteria and reporting guidelines in order for radiomics to mature as a discipline (see www.radiomics.world). Certain author's proposed that radiomics could be used as a "virtual biopsy". It could be the case in the sense that several reports demonstrated that biological features of tumours such as EGFR mutations, HPV status and even hypoxia could be quantified by radiomics (6). There are however two main differences: a) Radiomics is based on the whole tumour in contrast to a biopsy taken most often randomly in an heterogeneous tumour and b) the radiomics values is a continuous variable in contrast to molecular biology assays which are often dichotomized (e.g. $\mathrm{mt}$ vs wt). Interestingly, certain radiomics signatures e.g. a proliferation radiomics signature, works as well with cone beam CT which opens the field of "4D-Radiomics" $(4,7)$.

Conclusions. There is a need of preclinical Radiomics studies to establish causal relationship between biology and image signatures (3).

https://doi.org/10.1016/j.ejmp.2018.06.304

[I233] Automated treatment planning and quality assurance Crister Ceberg ${ }^{\mathrm{a},{ }^{*}}$, Hunor-Jeno Benedek ${ }^{\mathrm{b}}$, Tommy Knöös ${ }^{\mathrm{b}}$

${ }^{a}$ Lund University, Medical Radiation Physics, Lund, Sweden

${ }^{\mathrm{b}}$ Lund University Department of Clinical Sciences, Medical Radiation Physics, Skåne University Hospital Department of Hematology, Oncology and Radiation Physics, Lund, Sweden

* Corresponding author.

In radiotherapy, there is a strong development striving at improving the quality of the treatment plans. Consequently, there is a great need for quality assurance procedures focusing on the treatment plan quality. The most fundamental aspect is perhaps the quality of a treatment plan for an individual patient (treatment plan optimization), which in turn is dependent on the quality of the underlying protocols and prioritization strategies (guidelines). However, there are also other important aspects, such as quality assurance of treatment planning algorithms and their application to specific diagnoses or treatment techniques (benchmarking), preparatory treatment planning for multi-centre clinical studies (dummy runs), and treatment planner achievements (training and competencies). The quality of a treatment plan may also encompass its deliverability at treatment (QC pass rate and plan robustness). To approach these issues in a quantitative fashion, there must be an unambiguous understanding of the concept of treatment plan quality, that can be applied in a continuous quality assurance program. While the plan quality in its fundamental sense is related to the degree of prescription fulfilment, it is also influenced by various limitations and uncertainties. In this respect, it is of foremost importance to use optimization objectives that are specific and measurable. Many of these issues can be addressed using automatically generated treatment plans, either data-base driven or protocol based, and extended to sets of multiple Pareto-optimal plans. In this presentation, we will review some of the possibilities for treatment plan quality assurance that becomes available with automated treatment planning techniques. The influence of short-cuts and limitations will be discussed.

https://doi.org/10.1016/j.ejmp.2018.06.305 